\section{PERCEPÇÃO E UTILIZAÇÃO DA EDUCAÇÃO EM SAÚDE PARA O USO RACIONAL DE MEDICAMENTOS POR MÉDICOS}

\author{
Perception and use of health education for rational use of \\ drugs by physicians
Percepción y utilización de la educación para la salud en el uso racional de medicamentos por los médicos

\section{RESUMO}

Objetivo: Compreender a percepção e utilização da educação em saúde para o uso racional de medicamentos (URM) pelos médicos inseridos na atenção primária em saúde. Métodos: Tratou-se de pesquisa qualitativa, descritiva e exploratória, realizada em 2011, com sete médicos da Estratégia de Saúde da Família, por meio de entrevista individual semiestruturada. Dados foram analisados pela técnica de análise do conteúdo, emergindo três categorias temáticas e suas subcategorias: percepção do conceito de uso racional de medicamentos de forma parcial; percepção do conceito de educação em saúde de forma parcial; não valorização da potencialidade da utilização da educação em saúde no uso racional de medicamento. Resultados: Os resultados evidenciaram que a percepção dos médicos é fragmentada e dividida em conceitos relacionados aos profissionais, à enfermidade, ao medicamento e ao paciente e comunidade. Conclusão: A utilização da educação em saúde pelos médicos prioriza o modelo tradicional de educação em saúde, privilegiando a transmissão do conhecimento técnico-científico.

Descritores: Educação em Saúde; Uso de Medicamentos; Saúde da Família; Pesquisa Qualitativa.

\section{ABSTRACT}

Objective: To understand the perception and utilization of health education for the rational use of medicines (RUM) by physicians working in primary healthcare. Methods: Qualitative, descriptive, and exploratory research, conducted in 2011, with seven physicians of the Family Health Strategy, by means of individual semi-structured interview. Data was analysed through the content analysis technique, emerging three thematic categories and their subcategories: partial perception of the concept of rational medicines use; partial perception of the concept of health education; not valuing the potentiality of employing health education in rational medicines use. Results: The results showed that the physicians' perception of the concepts are fragmented and divided into concepts related to the professionals, the disease, the drug, and to the patient and community. Conclusion: The use of health education by the physicians prioritizes its traditional model, with emphasis on the transmission of technical-scientific knowledge.

Descriptors: Health Education; Drug Utilization; Family Health; Qualitative Research.

\section{RESUMEN}

Objetivo: Comprender la percepción y utilización de la educación para la salud en el uso racional de medicamentos (URM) por los médicos de la atención primaria en salud. Métodos: Se trató de una investigación cualitativa, descriptiva y exploratoria realizada en 2011 con siete médicos de la Salud de la Familia a través de una entrevista semiestructurada individual. Los datos fueron analizados con la técnica de análisis de contenido, surgiendo tres categorías temáticas y sus subcategorías: la percepción del concepto de uso racional de medicamentos de forma parcial; la percepción del concepto de educación para la salud de
Artigo Original

1) Universidade Estadual de Montes Claros - UNIMONTES - Montes Claros (MG) Brasil

2) Universidade Federal de Minas Gerais UFMG - Belo Horizonte (MG) - Brasil 
forma parcial; la no valoración de la potencialidad de la utilización de la educación para la salud en el uso racional de medicamento. Resultados: Los resultados evidenciaron que la percepción de los médicos está fragmentada y dividida en conceptos relacionados a los profesionales, la enfermedad, los medicamentos, al paciente y la comunidad. Conclusión: La utilización de la educación para la salud por los médicos prioriza el modelo tradicional de la educación en salud favoreciendo la transmisión del conocimiento técnico-cientifico.

Descriptores: Educación en Salud; Utilización de Medicamentos; Salud de la Familia; Investigación Cualitativa.

\section{INTRODUÇÃO}

Em todo o mundo, mais de $50 \%$ dos medicamentos são receitados, dispensados e vendidos de forma inadequada ${ }^{(1)}$. Essa prática irracional não é exclusiva do Brasil, sendo considerada um importante problema de saúde pública mundial, com grandes consequências econômicas ${ }^{(2)}$. O simbolismo de que se revestem os medicamentos na sociedade tem contribuído para o seu uso irracional. A propaganda massiva e a facilidade de acesso a medicamentos em farmácias fazem crer que estes são produtos livres de riscos. Além disso, a baixa frequência de textos tratando dos riscos de utilização de medicamentos tende a reforçar o mito da saúde promovido pelo uso deles e o caráter simbólico do seu poder de cura $^{(3,4)}$.

A educação em saúde consiste no campo de teorias e práticas que se ocupa das relações entre o conhecimento e os processos de saúde e doença dos indivíduos e da coletividade $^{(5)}$. Essa construção de conhecimento é transversalizada por um possível diálogo entre o saber instituído, elaborado pela produção científica e sujeito a uma revisão permanente, e o senso comum, resultante da vivência cotidiana e baseado em relações perceptivas e afetivas, de significados próprios ${ }^{(5)}$.

No entanto, esses aspectos não são suficientes para transformar o cotidiano do usuário, já que a pedagogia utilizada tem papel fundamental, sendo a problematizadora capaz de fazê-la ${ }^{(6)}$.

O desenvolvimento de atividades educacionais de caráter público constitui um dos meios de alcançar o uso racional de medicamentos, proposto pela Organização Mundial de Saúde (OMS) ${ }^{(1)}$, que reconhece o farmacêutico como o profissional mais capacitado para esse trabalho, inclusive prestando assessoramento técnico aos demais profissionais de saúde ${ }^{(7)}$. Estabeleceu também como seu grande desafio para a próxima década a melhoria na racionalidade do uso de medicamentos, havendo uma necessidade de promover a avaliação desse uso e vigiar o seu consumo ${ }^{(1)}$.

As estratégias para promover o uso racional de medicamentos distribuem-se segundo o público-alvo que se quer sensibilizar, sendo categorizadas como estratégias educacionais, gerenciais e regulatórias ${ }^{(2)}$. Novas estratégias educacionais e gerenciais para lidar com a educação permanente dos médicos são concebidas e implantadas ${ }^{(8)}$.

Estudos em grandes organizações e, em menor grau, no sistema de saúde apontam consistentemente para a importância das redes informais e das comunidades profissionais para trazer grandes mudanças ${ }^{(9)}$. Um dos instrumentos utilizadas durante as práticas educativas têm sido as comunidades de prática, que são as organizações que se definem pela partilha de uma prática entre os seus diferentes membros ${ }^{(10)}$.

No Brasil, o Ministério da Saúde tem proposto a Educação Permanente como uma estratégia de transformação das práticas de formação, atenção, gestão, formulação de políticas, participação popular e controle social no setor de saúde ${ }^{(11)}$. No estado de Minas Gerais, o Programa de Educação Permanente para os médicos da saúde da família tem como propósito induzir uma cultura de mudança e renovação da prática do profissional e criar um sistema de melhoria permanente da performance clínica, em busca da excelência dos cuidados ao paciente ${ }^{(8)}$.

Estudos que avaliam a educação em saúde na utilização dos medicamentos estão focados na apresentação de resultados que refletem as práticas encontradas, não se preocupando em indicar possíveis formas de atuação para reverter tais práticas, e os profissionais de saúde não se sentem suficientemente habilitados para desenvolver uma prática profissional mais próxima do usuário ${ }^{(12)}$.

Existe, portanto, a necessidade de se responder por que as ações de educação em saúde não estão atingindo os objetivos esperados. Estudar sobre essas questões justificase uma vez que são escassos os estudos qualitativos que abordem de forma sistemática as atividades educativas em saúde sob a ótica do médico, tanto na literatura nacional como internacional.

Dessa forma, o propósito deste trabalho é compreender a percepção e utilização da educação em saúde para o uso racional de medicamentos (URM) pelos médicos inseridos na atenção primária em saúde.

\section{MÉTODOS}

Tratou-se de pesquisa qualitativa, de caráter descritivo e exploratório, em cenários da atenção primária, utilizandose a Estratégia Saúde da Família (ESF), na cidade de Teófilo Otoni-MG, realizada nos meses de março e abril de 2011. 
Considerou-se suficiente o número de participantes investigados quando os dados da pesquisa se tornaram repetitivos, deixando de acrescentar novas informações ${ }^{(13)}$. O critério de inclusão utilizado era ser médico e estar inserido na ESF por um período superior a seis meses. O critério de exclusão ocorreu com a não aceitação em participar da pesquisa, totalizando sete participantes no presente estudo.

Realizou-se a coleta de dados no consultório médica da ESF, após a leitura dos objetivos da pesquisa e obtenção do Termo de Consentimento Livre e Esclarecido. A entrevista individual semiestruturada teve duas etapas, a primeira contemplando os dados de identificação dos entrevistados com as variáveis sociodemográficas e a segunda com as questões norteadoras relacionadas aos objetivos do estudo: "O que você entende sobre uso racional de medicamentos?", "Qual a importância das ações educativas para o uso correto de medicamentos", "Em algum momento das suas atividades profissionais você realiza ações educativas na promoção do URM? Onde? Como? Quais?", "Quais os profissionais mais indicados para este trabalho?" e "Como você vê a presença do farmacêutico nesta perspectiva?".

As entrevistas gravadas, com duração médica de 35 minutos, eram transcritas em seguida, permitindo contar integralmente com as informações fornecidas pelos participantes e possibilitando a fidedignidade dos dados.

Para a análise dos dados, utilizou-se a técnica de análise do conteúdo ${ }^{(14)}$, na modalidade temática, com as categorias não definidas a priori. Em um primeiro momento, realizouse a leitura flutuante das entrevistas, definindo-se as unidades de registro e de contexto. Posteriormente, definiram-se as categorias de análise, de modo que permitissem agrupar as unidades de registro e contexto com características comuns ou que se relacionavam entre si. Finalmente, procedeuse à interpretação dos dados, buscando desvendar o conteúdo subjacente ao que foi manifestado. $\mathrm{Na}$ análise dos resultados, as falas foram codificadas como M1, M2, M3, M4, M5, M6 e M7.

Emergiram da transcrição das gravações as seguintes categorias e subcategorias: "Percepção do conceito do uso racional de medicamentos de forma parcial", com suas subcategorias explorando conceitos relacionados aos profissionais, à enfermidade, ao medicamento, ao paciente e à comunidade; "Percepção do conceito de Educação em Saúde de forma parcial", sendo as subcategorias os conceitos relacionados aos profissionais, à enfermidade, ao medicamento e ao paciente e comunidade; "Não valorização da potencialidade da utilização da Educação em Saúde no URM", com suas subcategorias tratando de conceitos relacionados aos pacientes e comunidade e relacionadas aos profissionais.

A presente pesquisa está em conformidade com os princípios da Declaração de Helsinque, tendo sido aprovada pelo Comitê de Ética em Pesquisa da Universidade Estadual de Montes Claros (UNIMONTES), sob parecer nº 2051/10.

\section{RESULTADOS E DISCUSSÃO}

Entrevistaram-se sete médicos: cinco do sexo masculino e dois do sexo feminino; quatro casados, dois separados e um solteiro; com idade entre 27 e 41 anos.

O tempo de trabalho na ESF variou de um ano e meio a 13 anos, mas apenas um médico possuía especialização em Saúde da Família; os demais possuíam outras especializações (dermatologia, geriatria, endocrinologia, cirurgia plástica e infectologia). Esse dado confirma a realidade do Brasil, onde a maioria dos médicos incluídos no Sistema Único de Saúde (SUS) é generalista ${ }^{(15)}$.

No que se refere à educação permanente, apenas um médico participou de capacitação sobre o uso racional de medicamentos (URM). Os demais só estudaram sobre o tema durante a graduação médica, o que está em desacordo com as orientações da OMS, que aponta a educação formal e permanente em farmacoterapia da equipe de saúde ${ }^{(1)}$

A seguir, apresentam-se as categorias temáticas e suas subcategorias, com as falas dos médicos entrevistados.

\section{"Percepção do conceito de uso racional de medicamentos de forma parcial"}

Esta categoria emergiu do conceito proposto pela OMS sobre o URM, que define: "O paciente recebe o medicamento apropriado a sua necessidade clínica, na dose e posologia corretas, por um período de tempo adequado e ao menor custo para si e para a comunidade ${ }^{(1)}$ ".

Percebeu-se que os médicos têm conceitos fragmentados, um completando o outro, mas de maneira geral, não de forma integral, podendo dividi-los com conceitos relacionados aos profissionais, à enfermidade, ao medicamento, ao paciente e à comunidade.

\section{Conceitos relacionados aos profissionais}

AOMS propõeque, para ouso racional demedicamentos, é preciso, inicialmente, estabelecer a necessidade do uso, receitando o medicamento apropriado, de acordo com os ditames de eficácia e segurança comprovados e aceitáveis ${ }^{(1)}$. Nessa perspectiva, o conhecimento dos médicos, assim como as habilidades e atitudes relacionadas à prescrição são fatores importantes na prescrição dos medicamentos.

"URM é você prescrever o medicamento de acordo com a indicação." (M2)

"Se tratar o paciente, aquela é melhor, a outra é melhor." (M1)

"Eu tenho uma mão leve ao prescrever medicações." (M6) 
O uso racional de um medicamento começa pela qualidade do produto que se está administrando, passando pela indicação adequada e a posologia ideal ${ }^{(16)}$. No entanto, o aumento da complexidade da prescrição suscetibiliza o indivíduo a interações medicamentosas, possíveis reações adversas, surgimento de comorbidades e, consequentemente, diminuição da sua qualidade de vida ${ }^{(17)}$.

\section{Conceitos relacionados com a enfermidade}

$\mathrm{O}$ medicamento deve ser apropriado às necessidades clínicas $^{(1)}$, e o prescritor é o responsável pela indicação de um medicamento com base na interpretação que faz daquilo que o paciente lhe $\mathrm{diz}^{(18)}$.

"Prescrever de acordo com indicação precisa da patologia que o paciente tem.” (M2)

"Por que usar e em que patologia deve estar sendo usado.” (M5)

Percebe-se, pelas falas, que o conhecimento dos profissionais estabelece claramente a fronteira entre o normal e o patológico, traduzida em um padrão que fundamenta diagnósticos e intervenções ${ }^{(19)}$.

"É o uso adequado pra certas doenças [...] e que a doença tem que ser tratada." (M1).

Nesse primeiro momento, os relatos não trazem o enfoque da promoção de saúde, defendida na Carta de Ottawa com a visão positiva da saúde, identificando-a com bem-estar e qualidade de vida, e não simplesmente com a ausência de doença ${ }^{(20)}$.

\section{Conceitos relacionados ao medicamento}

Reconhecidos como instrumentos indispensáveis às ações de saúde, os medicamentos ocupam posição central na terapêutica ${ }^{(4)}$. Em farmacologia, é sempre preciso analisar o custo-benefício do uso de cada medicamento e a possibilidade de respostas iatrogênicas. Para tanto, são necessários conhecimentos em farmacologia, bioquímica, fisiologia, patologia, microbiologia e conhecimentos mínimos de interação medicamentosa ${ }^{(21)}$. A indicação terapêutica está intimamente relacionada a um diagnóstico preciso $^{(16)}$. Já a posologia leva em conta o diagnóstico e as características individuais do usuário ${ }^{(16)}$, conforme descritas nestas colocações:

"Prescrever o medicamento no tempo certo, na dosagem certa." (MI)

"Todo medicamento tem seu efeito benéfico e seu efeito deletério." (M4)

"O que tem de melhor, tem que levar em conta: a ação, os fatores de resistência..." (M5)
Dessa forma, é necessário que o medicamento seja prescrito adequadamente na forma farmacêutica, constando doses e período de duração do tratamento ${ }^{(1)}$. E com a correta utilização, para o alcance dos objetivos terapêuticos ${ }^{(22)}$.

\section{Conceitos relacionados ao paciente e comunidade}

$\mathrm{O}$ acesso aos medicamentos essenciais à saúde da população é o primeiro passo para o estabelecimento de uma política de medicamentos ${ }^{(18)}$. É importante que estejam disponíveis de modo oportuno ${ }^{(1)}$.

"A gente sabe que o que adianta a gente fechar um diagnóstico, passar um medicamento, eu posso até considerar ele adequado, mas o paciente não usar." (M7)

É importante também que tenha um preço acessível ${ }^{(1)}$, uma vez que pode influenciar a utilização do restante do cuidado médico ${ }^{(18)}$.

"O que tem de melhor, tem que levar em conta o custobeneficio." (M5)

"Eu acho que o URM é você prescrever o medicamento avaliando o custo-beneficio." (M4)

E que responda sempre aos critérios de qualidade exigidos; que se dispense em condições adequadas, com a necessária orientação e responsabilidade ${ }^{(1)}$.

"Uso racional eu entendo como uso controlado dentro das orientações que são feitas pelo médico no momento da prescrição da medicação." (M3)

E que se cumpra o regime terapêutico já prescrito, da melhor maneira possível ${ }^{(1)}$ :

"Se o paciente teve uma boa resposta ou não." (M1)

O uso adequado dos medicamentos não depende apenas de uma prescrição de qualidade, mas também de uma dispensação responsável ${ }^{(18)}$. Percebeu-se, com as falas contidas nesta subcategoria, a participação do prescritor e do paciente, não sendo reconhecido o papel do dispensador, responsável pela dispensação correta, incluindo informações apropriadas sobre os medicamentos prescritos. A interação entre médico, farmacêutico e paciente possibilita o surgimento de expectativas, demandas e trocas de informações que terão consequências diretas no resultado da terpêutica ${ }^{(18)}$.

Os requisitos para a promoção do URM são muito mais complexos e envolvem uma série de variáveis. Para que sejam cumpridos, devem contar com a participação de diversos atores sociais: pacientes, profissionais de saúde, legisladores, formuladores de políticas públicas, indústria, comércio e governo ${ }^{(23)}$. 


\section{“Percepção do conceito de Educação em Saúde de forma parcial"}

Esta categoria reúne elementos que identificam as concepções de educação em saúde pelos médicos da Estratégia Saúde da Família entrevistados no presente estudo, percebendo-se que também possuem conceitos fragmentados sobre educação em saúde, podendo dividi-los em conceitos relacionados aos profissionais, à enfermidade, ao medicamento e ao paciente e comunidade.

\section{Educação em saúde relacionada ao profissional}

Dentre as intervenções fundamentais para promover o URM, destacam-se a educação médica continuada como requisito para o desempenho da profissão e a educação da população sobre medicamentos ${ }^{(1)}$.

A Educação Permanente em Saúde é uma estratégia de transformação das práticas em saúde, sendo a adesão do profissional médico um dos desafios para sua efetivação ${ }^{(24)}$, visto que a necessidade de aprendizagem do médico é diária. Para o atendimento médico, podem surgir dúvidas que, se dissipadas antes de qualquer tomada de decisão, trariam benefícios para ele e seu paciente. É um processo cotidiano. Não se faz a partir de eventos periódicos; é uma educação permanente ${ }^{(8)}$.

"Eu faço isso comigo. Uma vez por mês eu elejo um tema." (M5)

Não há dúvidas de que os riscos associados à terapêutica podem ser minimizados se houver consciência dos profissionais da saúde em melhorar a qualidade das informações ou discutir alternativas de tratamento para resolver a queixa do paciente ${ }^{(12)}$.

As falas a seguir refletem a especialidade do profissional nas ações de educação em saúde:

"Dentro da minha formação de geriatria, a gente tem que conhecer profundamente tanto patologias quanto efeitos colaterais dos medicamentos [...]. A minha conduta é sempre uma conduta geriátrica." (M6)

A presença de especialistas trabalhando como generalistas no serviço prejudicam o processo de assistência, uma vez que a fragmentação do conhecimento dificulta a integralidade das ações em saúde, sendo necessário que haja mudanças em ambos os cenários, tanto na formação como nos serviços de saúde, para que ocorram mudanças efetivas no paradigma assistencial ${ }^{(15)}$.

\section{Educação em saúde relacionada com a enfermidade}

A forma como a população compreende e conceitua as doenças e os tratamentos é um dos fatores que influenciam o uso de medicamentos e a adesão ao tratamento. Os fatores culturais influenciam na percepção e determinam quais sinais e sintomas são percebidos como "anormais" e originam um padrão de adoecimento para o doente e para aqueles que o cercam $^{(25)}$. A modificação de postura dos sujeitos investigados em um estudo(26) esteve ligada a um processo de (re)conhecimento da doença, com base em suas representações e experiências com a enfermidade ${ }^{(26)}$. É importante que a educação em saúde busque explorar a forma como a doença é elaborada culturalmente, buscando levar o sujeito ao reconhecimento desta como algo anômalo, retirando a visão de ser normal, natural ${ }^{(26)}$.

Pode-se observar que os médicos possuem uma visão curativista, com foco nas patologias, apresentando uma relação profissional-paciente impositiva, de acordo com o modelo tradicional da educação em saúde. Não conseguem se despir do rigor do conhecimento científico voltado para a prevenção, para o biológico.

"Qualquer dorzinha de cabeça não precisa usar antibióticos [...], sempre avaliar o meio onde se vive pra evitar certas doenças.” (M1)

"Oriento que infecção é diferente de inflamação." (M3)

O modo de organização pautada nesse modelo biomédico, no qual as ações são majoritariamente pensadas para as doenças, e não para os doentes, estabelece barreiras tanto para o usuário quanto para o profissional desenvolver ações em saúde ${ }^{(25)}$.

\section{Educação em saúde relacionada ao medicamento}

É necessário que a população esteja orientada sobre como proceder em relação ao uso de medicamentos, para que estes tenham uma ação segura e se diminuam os riscos de efeitos colaterais ou adversos e de interações medicamentosas ${ }^{(23)}$. A educação acerca do uso de medicamentos está envolvida em grande parte das atividades relacionadas com o estabelecimento e manutenção da saúde do indivíduo:

"Ação educativa previne o uso abusivo de determinadas drogas [...] e previne também até os efeitos colaterais das associações entre elas.” (M6)

"Sempre que possível, eu procuro estar orientando pra que serve o medicamento." (M4)

Faz-se necessário que a sociedade se conscientize e entenda que o mesmo medicamento que cura pode matar ou deixar danos irreversíveis ${ }^{(23)}$. Observou-se a importância do profissional em ressaltar também os pontos negativos dos medicamentos, como os efeitos transitórios, os efeitos adversos e os possíveis efeitos rebotes. 
"Eu abordo a necessidade de tomar o tempo necessário, a quantidade necessária, eu explico por que, da resistência." (M5)

\section{Educação em saúde relacionada ao paciente e à comunidade}

O doente e o público devem ter acesso à informação correta, objetiva e relevante a respeito dos medicamentos, para que se obtenha maior adequação em seu uso, além de evitar a automedicação desnecessária ${ }^{(4)}$. Foi constatado em uma pesquisa ser uma prática frequente em crianças, realizada por seus responsáveis ${ }^{(27)}$.

A busca pela conscientização da população quanto aos perigos da automedicação pelos profissionais de saúde está presente na maioria das entrevistas, visto que é uma prática frequente, sendo geralmente influenciada por amigos, familiares e balconistas de farmácias.

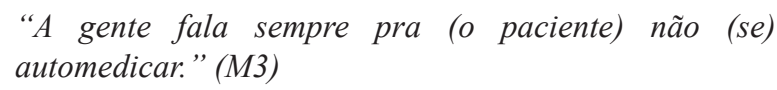

A automedicação é entendida como a prática de ingerir medicamentos sem o aconselhamento ou acompanhamento de um profissional de saúde qualificado ${ }^{(28)}$.

A adesão ao tratamento é complexa e envolve vários fatores, como características do usuário, características da doença, costumes culturais e de vida, tratamento medicamentoso (efeitos indesejáveis, esquemas complexos) e dificuldades institucionais. Um dos fatores decisivos é a confiança depositada na equipe de saúde, na prescrição e no médico $^{(25) \text {. }}$

"Dar liberdade para o paciente não ficar constrangido de retornar pra buscar, entendeu? Uma nova abordagem terapêutica, baseada em confiança, lógico!" (M4)

A educação em saúde, como prática social, traz implícita a visão cultural baseada em valores, crenças e visões de mundo, situados em tempo e espaço delimitados ${ }^{(29)}$.

"É claro que isso é um trabalho de longo tempo, pois é mudança de hábito, mudança de cultura." (M3)

Os trabalhadores devem refletir sobre diferenças culturais e atuar na perspectiva da educação humanizadora, crítica, reflexiva, voltada para a formação do homem integral e autônomo ${ }^{(29)}$.

\section{"Não valorização da potencialidade da utilização da Educação em Saúde no URM"}

Esta categoria se propõe a identificar as formas de utilização da Educação em Saúde sobre medicamentos pelos médicos da ESF investigados. Percebeu-se uma série de problemas e desafios na prática e limitações na utilização da educação em saúde, com certa ingenuidade na transferência de conhecimentos. Foram identificadas utilizações de educação em saúde relacionadas aos pacientes e comunidade, e relacionadas aos profissionais.

\section{Utilização da educação em saúde relacionada aos pacientes e comunidade}

Ao questionar as práticas de educação em saúde desenvolvidas pelos médicos, observou-se que eles as realizam apenas durante a consulta médica, limitando-se às orientações ao paciente.

\section{"Eu uso de paciente para paciente, só durante a consulta.” (M1) \\ "Eu faço ação educativa durante a consulta médica." (M6) \\ "Não há um treinamento especifico. A orientação é dada no momento da consulta.” (M3)}

As atividades de educação em saúde para os médicos na atenção básica ainda se concentram na abordagem diagnóstica e terapêutica, com poucas oportunidades de reflexão crítica das diretrizes do programa ${ }^{(5)}$.

Mudar a prática profissional de um médico é um processo complexo, visto que a aquisição de novos conhecimentos, habilidade e atitudes são insuficientes para empreender uma nova prática. Quando os médicos tomam conhecimento e compreendem as novas evidências, podem mudar sua prática e melhorar o cuidado aos seus pacientes $^{(8)}$. O conhecimento e a aprendizagem se tornaram os novos imperativos estratégicos das organizações ${ }^{(30)}$, sendo o conhecimento, e não simplesmente a informação, a principal fonte de potencial de uma organização ${ }^{(31)}$.

Nessa perspectiva, o termo "gestão do conhecimento" é entendido como um conjunto de processos que orienta a criação, disseminação e utilização do conhecimento para atingir plenamente os objetivos de uma organização, sendo efetivo somente quando há identidade coletiva e a existência de uma ampla rede social, sendo as redes humanas um dos veículos-chave para o compartilhamento de conhecimentos ${ }^{(9)}$. Um exemplo dessas redes são as Comunidades de Práticas (COP), que reúnem pessoas informalmente, por meio de interesses comuns ao aprendizado e sua aplicação. Em uma comunidade de prática, o conhecimento é construído à medida que indivíduos compartilham ideias através de mecanismos colaborativos, como narração e trabalho coletivo ${ }^{(9)}$.

$\mathrm{Na}$ maioria das vezes, a produção e a transmissão de informações a respeito dos medicamentos não se traduzem necessariamente em conhecimento $^{(18)}$. Então, o diálogo deve ser central na postura do profissional de saúde, o qual 
precisa aprender a ouvir e respeitar, ao mesmo tempo em que deve expor o que sabe a respeito de determinado tema. Dessa forma, profissionais e usuários podem construir, de forma compartilhada, um saber sobre o processo saúdedoença ${ }^{(32)}$.

"Eu tento fazer me esforçar ao máximo para que aquele paciente analfabeto saia com a informação dentro da cabeça, uma vez que eles são analfabetos..." (M2)

As orientações sobre medicamentos fornecidas aos pacientes são fundamentais para o sucesso do tratamento, uma vez que a ausência delas é uma das principais causas do uso incorreto de medicamentos ${ }^{(22)}$. Entretanto, há profissionais que não orientam os pacientes devido à falta de tempo.

"[...] Muito pouco, devido à falta de tempo. A gente usa, mas não chega a informar o paciente." (M7)

Nessa perspectiva, é preciso que sejam aprimorados os meios de fornecer a informação ao paciente. Isso envolve a formação dos profissionais, a reorganização do tempo dedicado à orientação pelo prescritor, a atuação direta do farmacêutico na dispensação dos medicamentos, bem como a organização dos serviços, para que essas informações possam ser repassadas ao paciente ${ }^{(22)}$.

Segundo a OMS, o excesso de trabalho desses profissionais é um dos principais motivos que contribuem para o uso irracional de medicamentos ${ }^{(1)}$.

Um estudo realizado sobre o perfil dos profissionais na Atenção Primária, na cidade de Montes Claros-MG, observou que, em relação à disponibilidade para dedicação exclusiva, $22,3 \%$ dos profissionais desempenham atividades em outras instituições, acumulando outras atividades ${ }^{(33)}$.

Os profissionais investigados sugeriram a palestra como a principal ação de educação em saúde para ser desenvolvida na unidade.

“[...] Na forma de palestra, pois eles entendem muito quando a gente se reúne." (M1)

"Eu acho que a forma mais barata e mais otimizada seria a palestra." (M4)

"O que poderia ser feito seria incluir nas palestras orientações sobre o uso racional de medicamentos." (M6).

"Fazendo palestras, entregando panfletos para aqueles que sabem ler, mostrar para aqueles que não sabem. Acho que um guia informativo sobre a medicação mais usada." (M1)

A educação em saúde, mesmo sendo o foco principal da Estratégia Saúde da Família, ainda deixa muito a desejar, pois é realizada apenas no momento em que são efetuados outros atendimentos de saúde, principalmente voltados à doença, e não seria somente esse o objetivo do trabalho educativo $^{(34)}$.

O trabalho das equipes de Saúde da Família está fundamentado nos referenciais teóricos de vigilância e promoção da saúde, por isso, devem atuar planejando seu processo de trabalho não apenas para atender à demanda espontânea, mas especialmente a fim de desenvolver ações para pessoas que ainda não conhecem ou não frequentam o serviço de saúde ${ }^{(29)}$.

"Nós poderíamos estar trabalhando a população adstrita como um todo [...], fazer por microáreas e sensibilizá-los quanto ao uso de medicação." (M5).

A equipe também deveria se responsabilizar pela população adstrita em seu território, resgatando os vínculos de compromisso e corresponsabilidade entre ela e a população, dentro dos princípios do Sistema Único de Saúde (SUS) ${ }^{(29)}$.

Os resultados explanados nessa subcategoria constataram que a maioria dos profissionais da ESF desconhece os fundamentos da educação em saúde e realiza práticas educativas verticais, distanciando-se da proposta de promoção da saúde e não privilegiando a criação de vínculo entre profissionais e população.

\section{Utilização da educação em saúde relacionada aos profissionais}

Ao questionar quais os profissionais mais indicados para realizar esse trabalho, foi considerada relevante a participação de todos os profissionais que compõem a equipe.

"[...] Dentro da equipe do PSF, eu penso que todos, desde o médico, o enfermeiro e os ACS." (M6)

"Eu acho que ele [médico] deveria ir a campo, pelo seu poder de convencimento." (M5)

"Na equipe do PSF, acho que o médico e o enfermeiro, pois não temos os outros profissionais." (M7)

A educação em saúde é uma ferramenta importante para a promoção da saúde e garantia dos direitos humanos fundamentais. $\mathrm{O}$ enfermeiro realiza essa prática associada ao cuidado prestado em todas as etapas da vida do ser humano ${ }^{(35)}$. Acredita-se que, por meio da educação em saúde como método de ensino dialógico, na busca pela construção compartilhada de conhecimento, o enfermeiro pode aprender a respeitar e potencializar a autonomia do usuário na luta por melhores condições de saúde ${ }^{(32)}$ : 
"O enfermeiro é uma profissão de cuidados mais eminentemente de mudança de comportamento a nível de eixo educativo." (M5)

Geralmente, há um descompasso entre o que pensam e sentem usuários e profissionais de saúde. Nesse sentido, é importante o papel do agente comunitário na equipe, pois, por pertencer àquela comunidade, ele conhece os problemas, as necessidades de saúde e cultura da população local e as dificuldades para enfrentá-los, podendo atuar como facilitador da relação entre a equipe e a comunidade ${ }^{(29)}$.

“Os ACS são a voz do PSF na comunidade.” (M6)

"Na verdade, a gente precisa conseguir trazer ACS mais pra perto do paciente, né?" (M2)

Também foi considerada relevante a contribuição do farmacêutico na equipe como orientador e colaborador nas ações de educação em saúde:

"Eu acho o médico, o farmacêutico e o enfermeiro são as três únicas áreas que teriam condição técnica pra poder estar executando esse tipo de trabalho.” (M4)

"Seria interessante ter um farmacêutico com a gente, pra fazer essas orientações, pra dar mais um suporte, pra estar presente na equipe também." (M3)

"Seria importante justamente para orientar os ACS (farmacêutico).” (M2)

A percepção dos médicos nesses discursos reconhece a importância do farmacêutico na atenção primária e as suas possibilidades de contribuição. Na mesma perspectiva, a OMS reconhece o farmacêutico como o profissional mais capacitado para conduzir ações destinadas à melhoria do acesso e promoção do URM, inclusive prestando assessoramento técnico aos demais profissionais de saúde ${ }^{(7)}$

Porém, essa realidade não se faz presente, pois as atividades de educação em saúde quanto ao URM são muitas vezes consideradas ainda incipientes no serviço público de saúde.

Além disso, a assistência farmacêutica é frequentemente realizada com o objetivo primordial de garantir o abastecimento dos medicamentos nas unidades, mediante processos de seleção, programação, aquisição, armazenamento, prescrição e dispensação ${ }^{(36)}$. Como se pode perceber nas falas seguintes, os profissionais consideram importante a presença da farmácia na unidade.

"O paciente já sairia com a receita, pegaria a medicação e poderia pegar informações complementares com o farmacêutico sobre medicamentos. " (M4)

"Eu acho que deveria ter a farmácia. A farmácia com o farmacêutico." (M3)
"Eu acho até estranho aqui na nossa região não ter farmacêutico na unidade...Eu acho fundamental ter uma farmacinha básica." (M1)

O farmacêutico precisa promover o uso racional dos medicamentos e a educação terapêutica na ESF. O tratamento, assim, torna-se mais eficaz e capacita o usuário para saber lidar com os possíveis efeitos colaterais e interações medicamentosas, contribuindo para a adesão ao tratamento. Dessa maneira, é preciso considerar o potencial de contribuição do farmacêutico e efetivamente incorporá-lo às equipes de saúde da família nas mais variadas atividades, promovendo saúde e prevenindo agravos ${ }^{(37)}$.

A participação da equipe multiprofissional nas atividades de educação em saúde e a incorporação do farmacêutico nesta perspectiva devem acontecer com uma preparação prévia. A fim de que ocorra a incorporação de ideias e práticas corretas que passem a fazer parte do cotidiano das pessoas, de forma a atender suas reais necessidades, é importante que, primeiro, a educação propicie ideias, para que estas sejam incorporadas na prática, gerando, então, mudança de comportamento.

A adoção de estratégias de promoção do URM pode trazer vários benefícios, como eficácia, segurança, melhoria do padrão de atendimento, significativa redução de custos e de morbimortalidade, e qualidade de vida para a população, sendo, portanto, de grande relevância social e científica.

De uma forma geral, pode-se perceber que não há registro se os médicos da Saúde da Família de Teófilo Otoni-MG utilizam de forma efetiva a "educação em saúde" na contribuição para a promoção do uso racional de medicamentos. Considerando a necessidade do fortalecimento da capacitação da comunidade na utilização dos medicamentos, procura-se identificar se os médicos inseridos no Programa Saúde da Família trocam informações ou promovem discussão com a população e a equipe sobre medicamentos.

Pode-se considerar como limitação deste estudo o fato de a investigação ter sido realizada apenas em nível local, tornando-se necessárias investigações semelhantes em outros estados do Brasil para possíveis cotejamentos.

\section{CONSIDERAÇÕES FINAIS}

A percepção e utilização de educação em saúde pelos médicos neste estudo priorizam o modelo tradicional de educação em saúde, privilegiando a transmissão do conhecimento técnico-científico. Percebe-se que os conceitos são fragmentados; ausência de conhecimentos sobre o URM na formação prévia; utilização de metodologias aplicadas pontualmente e na perspectiva paternalista. Percebe-se, ainda, a preocupação com a prevenção das doenças, e não 
com a promoção da saúde; profissionais pouco disponíveis; e não reconhecimento das necessidades, em suas ações. Sugere-se que a educação em saúde seja realizada com capacitação nas metodologias, com o profissional preparado para fazê-la. E que a incorporação do farmacêutico na equipe, como sugerido pelos profissionais e recomendado pela OMS, aconteça com a incorporação de ideias e práticas que possibilitem uma mudança de comportamento.

Este trabalho pode contribuir para o debate sobre a transformação da prática educativa, de modo que as atividades de educação em saúde sejam entendidas não como uma atividade a mais a ser realizada, mas como um instrumento que reoriente o uso indiscriminado dos medicamentos. Nesse sentido, que se possa desmistificar o simbolismo com o qual se reveste o medicamento no processo saúde-doença, e que a consulta médica não se resuma apenas a uma prescrição medicamentosa. O que se propõe é uma mudança de atitude, sem necessariamente acrescentar mais uma atividade, pois é no cotidiano, na prática das atividades regulares, que se estabelece a ação educativa; mas é preciso que o profissional esteja apto e disposto a mudar as suas ações para o bem da população.

\section{REFERÊNCIAS}

1. Organización Mundial de la Salud. Perspectivas políticas sobre medicamentos de La OMS: promoción del uso racional de medicamentos-componentes centrales. Ginebra: OMS; 2002.

2. Ministério da Saúde (BR). Oficial de trabalho uso racional de medicamentos na perspectiva multiprofissional. Brasília: Organização PanAmericana da Saúde; 2007.

3. Pereira Júnior AC, Telles Filho PCP, Azevedo DSS. Automedicação: consumo, orientação e conhecimento entre acadêmicos de enfermagem. Rev Enferm UFPE. 2013;7(6):4472-8.

4. Lage EA, Freitas MIF, Acurcio FA. Informações sobre medicamentos na imprensa: uma contribuição para o uso racional? Ciênc Saude Coletiva. 2005;10(Supl):133-9.

5. Gazzinelli MFC. Representações sociais da educação em saúde pelos profissionais da equipe de saúde da família. Trab Educ Saúde. 2013;11(3):553-71.

6. Freire P. Pedagogia do oprimido. Rio de Janeiro: Paz e Terra; 2005.

7. Organización Mundial de la Salud. Punto 19 del orden del día. In: 47a Asamblea Mundial de la Salud; 1994. Ginebra: OMS; 1994.
8. Silvério JB. Programa de educação permanente para médicos de família. Rev Méd Minas Gerais. 2008;18(4Supl):60-6.

9. Bate SP, Robert G. Knowledge Management and communities of practice in the private sector: lessons for modernising the National Health Service in England and Wales. Public Admin. 2002;80(4):643-63.

10. Illera JLR. Como as comunidades virtuais de prática e de aprendizagem podem transformar a nossa concepção de educação. Rev Cienc Educ. 2007;(3):117-24.

11. Ministério da Saúde (BR), Secretaria de Gestão do Trabalho e da Educação na Saúde, Departamento de Gestão da Educação na Saúde. A educação permanente entra na roda: Polos de educação permanente em saúde: conceitos e caminhos a percorrer. Brasília: Ministério da Saúde; 2005.

12. Leite SN, Vieira M, Veber AP. Estudo de utilização de medicamentos: uma síntese de artigos publicados no Brasil e América Latina. Ciênc Saúde Coletiva. 2008;13(Supl):793-802.

13. Minayo MCS. O desafio do conhecimento: pesquisa qualitativa em saúde. São Paulo: Hucitec; 2007.

14. Bardin L. Análise de conteúdo. Lisboa: Edições 70; 1979.

15. Carácio FCC, Conterno LO, Oliveira MAC, Oliveira ACH, Marin MJS, Braccialli LAD. A experiência uma instituição pública na formação do profissional de saúde para atuação em atenção primária. Ciênc Saúde Coletiva. 2014;19(7):2133-42.

16. Lima GB, Nunes LCC, Barros JAC. Uso de medicamentos armazenados em domicílio em uma população atendida pelo Programa Saúde da Família. Ciênc Saúde Coletiva. 2010;15(Supl 3):3517-22.

17. Frohlich SE, Zaccolo AV, Silva SL, Mengue SS. Association between drug prescribing and quality of life in primary care. Pharm World Sci. 2010;32(6):74451.

18. Pepe VLE, Castro CGSO. A interação entre prescritores, dispensadores e paciente: informação compartilhada como possível benefício terapêutico. Cad Saúde Pública. 2000; 16(3):815-22.

19. Ferreira DC, Souza ID, Assis CRS, Ribeiro MS. A experiência do adoecer: uma discussão sobre saúde, doença e valores. Rev Bras Educ Méd. 2014;38(2): 283-8.

20. Buss PM, organizador. Promoção da Saúde e Saúde Pública: contribuição para o debate entre as escolas de 
saúde pública na América Latina. Rio de Janeiro; 1998. $178 \mathrm{p}$.

21. Silva FM, Goulart FC, Lazarini CA. Caracterização da prática de automedicação e fatores associados entre universitários do curso de Enfermagem. Rev Eletr Enferm. 2014;16(3):644-51.

22. Oenning D, Oliveira BV, Blatt CR. Conhecimento dos pacientes sobre os medicamentos prescritos após consulta médica e dispensação. Ciênc Saúde Coletiva. 2011;16(7):3277-83.

23. Aquino DS. Por que o uso racional de medicamentos deve ser uma prioridade? Ciênc Saúde Coletiva. 2008;13(Supl):733-6.

24. D’Ávila LS, Assis LN, Melo MB, Brant LC. Adesão ao Programa de Educação Permanente para médicos de família de um Estado da Região Sudeste do Brasil.Rio de Janeiro. Ciênc Saude Coletiva. 2014;9(2):401-16.

25. Coutinho FHP, Sousa IMC. Percepção dos indivíduos com hipertensão arterial sobre sua doença e adesão ao tratamento medicamentoso na estratégia saúde da família. Rev Baiana Saúde Pública. 2011;35(2):397411.

26. Gazzinelli MF, Gazzinelli A, Reis DC, Penna CMM. Educação em saúde: conhecimentos, representações sociais e experiência da doença. Cad Saúde Pública. 2005;21(1):200-6.

27. Beckhauser GC, Souza JM, Valgas C, Piovezan AP, Galato D. Utilização de medicamentos na Pediatria: a prática de automedicação em crianças por seus responsáveis. Rev Paul Pediatr. 2010;28(3):262-8.

28. Masson W, Furtado PL, Lazarini CA, Conterno LO. Automedicação entre acadêmicos do curso de medicina da Faculdade de Medicina de Marília, São Paulo. Rev Bras Pesq Saúde. 2012;14(4):82-9.

29. Alves GG, Aerts D. As práticas educativas em saúde e a Estratégia Saúde da Família. Ciênc Saúde Coletiva. 2011;16(1):319-25.

30. Allee V. Knowledge networks and communities of practice. OD Practitioner [periódico na Internet]. 2000 [acesso em 2014 Set 02];32(4):13. Disponível em: http:/www.vernaallee.com/images/vaa-knowledgenet worksandcommunitiesofpractice.pdf
31. Marshall L. Facilitating knowledge management and knowledge sharing: new opportunities for information professionals. Online Inc. 1997;21(5):92-98.

32. Silva LD, Beck CLC, Dissen CM, Tavares JP, Budó MLD, Silva HS. Enfermeiro e a educação em saúde: um estudo bibliográfico. Rev Enferm UFSM. 2012;2(2):412-9.

33. Costa SM, Prado MCM, Andrade TN, Araújo EPP, Silva Júnior WS, et al. Perfil do profissional de nível superior nas equipes da Estratégia Saúde da Família em Montes Claros, Minas Gerais, Brasil. Rev Bras Med Fam Comunidade. 2013;8(27):90-6.

34. Roecher S. Educação em saúde na estratégia saúde da família: o significado e a práxis dos enfermeiros. Esc Anna Nery Rev Enferm. 2011;15(4):701-9.

35. Oliveira RL, Santos MEA. Educação em saúde na estratégia saúde da família: conhecimentos e práticas do enfermeiro. Rev Enferm Integrada. 2011;4(2): 833-44.

36. Alonso FG. Uso racional de los medicamentos. Med Clin. 1990;94(16):628-32.

37. Costa EM, Rabelo ARM, Lima JG. Avaliação do papel do farmacêutico nas ações de promoção da saúde e prevenção de agravos na atenção primária. Rev Cienc Farm Básica 2014;35(1):81-8.

\section{Endereço do primeiro autor:}

Ludmila Soares Antunes Bernardi

Programa de Pós Graduação em Ciências da Saúde da UNIMONTES

Campus Universitário Professor Darcy Ribeiro

Bairro: Vila Mauricéia

CEP: 39401-089 - Montes Claros - MG - Brasil

E-mail: ludmilafarm@gmail.com

\section{Endereço para correspondência:}

Luís Paulo Souza e Souza

Universidade Federal de Minas Gerais - Escola de

Enfermagem

Avenida Alfredo Balena, 190

Bairro: Santa Efigênia

CEP: 30.130-100 - Belo Horizonte - MG - Brasil

E-mail: luis.pauloss@hotmail.com 